\title{
Lung ultrasound in systemic sclerosis: correlation with high- resolution computed tomography, pulmonary function tests and clinical variables of disease
}

\author{
Antonietta Gigante $^{1}$ - Filippo Rossi Fanelli ${ }^{1}$ Silvio Lucci $^{1} \cdot$ Giuseppe Barilaro $^{1}$ • \\ Silvia Quarta ${ }^{1}$ Biagio Barbano ${ }^{1}$ - Antonello Giovannetti ${ }^{1}$ - Antonio Amoroso ${ }^{1}$. \\ Edoardo Rosato ${ }^{1}$
}

Received: 8 July 2015 / Accepted: 29 September 2015/Published online: 22 October 2015

(C) SIMI 2015

\begin{abstract}
Interstitial lung disease (ILD) is a hallmark of systemic sclerosis (SSc). Although high-resolution computed tomography (HRCT) is the gold standard to diagnose ILD, recently lung ultrasound (LUS) has emerged in SSc patients as a new promising technique for the ILD evaluation, noninvasive and radiation-free. The aim of this study was to evaluate if there is a correlation between LUS, chest HRCT, pulmonary function tests findings and clinical variables of the disease. Thirty-nine patients (33 women and 6 men; mean age $51 \pm 15.2$ years) underwent clinical examination, HRCT, pulmonary function tests and LUS for detection of B-lines. A positive correlation exists between the number of B-lines and the HRCT score $(r=0.81$, $p<0.0001$ ), conversely a negative correlation exists between the number of B-lines and diffusing capacity of the lung for carbon monoxide (DLCO) $(r=-0.63$, $p<0.0001)$. The number of B-lines increases along with the progression of the capillaroscopic damage. A statistically significant difference in the number of B-lines was found between patients with and without digital ulcers [42 (3-84) vs 16 (4-55)]. We found that the number of B-lines increased with the progression of both HRCT score and digital vascular damage. LUS may therefore, be a useful tool to determine the best timing for HRCT execution, thus, preventing for many patients a continuous and useless exposure to ionizing radiation.
\end{abstract}

Antonietta Gigante

antonietta_gigante@yahoo.it

1 Clinical Immunology Unit, Department of Clinical Medicine, Sapienza University of Rome, Viale dell'Università 37, 00185 Rome, Italy
Keywords Systemic sclerosis - Lung ultrasound · Interstitial lung diseases $\cdot$ High-resolution CT

\section{Introduction}

Interstitial lung disease (ILD) is a hallmark in the course of systemic sclerosis ( $\mathrm{SSc}$ ) and pulmonary fibrosis represents one of the main causes of morbidity and mortality [1]. High-resolution computed tomography (HRCT) is the gold standard for the diagnosis of ILD, and correlates with pulmonary function tests abnormalities [2, 3]. Recently, lung ultrasound (LUS) has emerged in SSc patients as a new promising technique for the evaluation of different pulmonary abnormalities, noninvasive and radiation-free. ILD can be suggested by the presence of B-lines (also called US lung comets) in LUS examination. B-lines originate from thickened interlobular septa, and are a sonographic sign of interstitial disease [4].

The aim of this study was to evaluate if there is a correlation between LUS, chest HRCT and pulmonary function tests findings. We also evaluated the correlation between LUS findings and the clinical variables of the disease: subset and duration of disease, modified Rodnan skin score (mRSS), disease activity index, disease severity score, digital ulcers history and capillaroscopic pattern.

\section{Materials and methods}

Thirty-nine patients (33 women and 6 men; mean age $51 \pm 15.2$ years) fulfilling the American College of Rheumatology/European League Against Rheumatism Collaborative Initiative criteria for classification and diagnosis of SSc, were enrolled in this study from 
Table 1 Epidemiological and clinical features of 39 patients with systemic sclerosis

\begin{tabular}{ll}
\hline Gender (female/male) & $33 / 6$ \\
Age, years & $51 \pm 15.2$ \\
Disease duration, years & $8.5 \pm 6.3$ \\
DAI & $2.4 \pm 1.5$ \\
DSS & $6.5 \pm 3.7$ \\
dcSSc/lcSSc & $24 / 15$ \\
SSc-specific autoantibodies, $n(\%)$ & \\
Anti-topoisomerase I & $23(59)$ \\
Anticentromere & $10(25.6)$ \\
None & $6(15.4)$ \\
Capillaroscopic pattern, $n(\%)$ & \\
Early & $12(30.8)$ \\
Active & $12(30.8)$ \\
Late & $15(38.5)$
\end{tabular}

$D A I$ disease activity score, DSS disease severity scale, $l c S S c$ limited cutaneous $\mathrm{SSc}, d c S S c$ diffuse cutaneous $\mathrm{SSc}$

November 2014 to April 2015 [5]. Fifteen patients had limited cutaneous SSc (lcSSc) and 24 presented diffuse cutaneous SSc (dcSSc) as defined by Le Roy et al. [6]. Table 1 shows the SSc patients' epidemiological and clinical features.

All SSc patients who had a need to undergo HRCT for staging or follow-up were enrolled. LUS examination for specific assessment of B-lines was performed within 4 weeks of the chest HRCT scan.

Patients with cardiac failure or valve diseases, idiopathic pulmonary fibrosis, venous occlusive disease (PVOD), chronic obstructive pulmonary diseases (COPD) were excluded. Smokers were also excluded.

The subjects' written consent was obtained according to the Declaration of Helsinki and the study has been approved by the ethics committee of Sapienza University.

\section{Lung ultrasound (LUS)}

LUS was performed by a Toshiba's Ultrasound System (Tokyo, Japan) equipped with a convex $2.5-$ to $3.5-\mathrm{MHz}$ probe. Although there is no clear consensus about lung ultrasound methodology, we used the methodology applied in previous studies of SSc patients.

We analyzed the anterior and lateral hemithoraces, scanning along the parasternal, midclavicular, anterior axillary, and midaxillary lines, to evaluate location and number of B-lines. A B-line was defined as a discrete laserlike vertical hyperechoic reverberation artifact that arises from the pleural line extending to the bottom of the screen without fading, moving synchronously with lung sliding. The number of B-lines was recorded for each intercostal space. The sum of B-lines yielded a score suggestive for the extent of ILD. A scan was considered positive either when $\geq 3$ B-lines were present in at least two adjacent scanning sites or when a total of $>5$ B-lines were recorded. A score of zero was defined as a complete absence of B-lines in all scanning sites. A full white screen in a single scanning site was scored as corresponding to $10 \mathrm{~B}$-lines [7, 8]. One observer acquired and analyzed all chest sonography studies. The intra-observer variability in B-line assessment was $3.8 \%$.

\section{Chest HRCT}

All HRCT examinations were performed according to standard protocol using a CT 64GE light Speed VCT power scanner with a rotation tube scanning time of $0.65 \mathrm{~s}$. Scans were obtained at full inspiration from the apex to the lung base with patients in supine position, at $120 \mathrm{kV}$ and $300 \mathrm{~mA} \mathrm{~s}$, and slice thickness and scans spacing of 1.25 and $7 \mathrm{~mm}$, respectively. HRCT examination did not include the use of contrast media agents. Parenchymal abnormalities on HRCT were coded and scored in all the images by independent readers, blinded with respect to LUS results, according to the score elaborated by Warrick et al. This score includes a 'severity score', ranging from 0 (normal) to 15 (all lesions present), and an 'extension score', ranging from 0 (normal) to 15 (more than nine pulmonary segments involved). A total Warrick score was obtained by summing the severity and the extension scores [9].

\section{Baseline pulmonary function tests (PFT)}

Spirometric parameters of flows and volumes [(FEV1, forced expiratory volume in the 1st second), FVC (forced vital capacity), FEV1/FVC)] and single-breath carbon monoxide (CO) diffusing capacity (DLCO), corrected for hemoglobin concentration, were recorded with a Quark PFT 2 spirometer (Cosmed) and expressed according to the standards recommended by the American/European Respiratory Society [10]. All spirometric parameters are expressed as percentage of predicted.

\section{Nailfold videocapillaroscopy}

Nailfold videocapillaroscopy (NVC) was performed by an optical probe, equipped with magnification $200 \times$ contact lens and connected to an image analysis software (Pinnacle Studio Version 8). The identified patterns were classified as early, active or late [11].

\section{Skin thickening, disease activity and disease severity}

Skin thickening was assessed by a mRSS, which is the most used method to assess skin induration in SSc. It is 


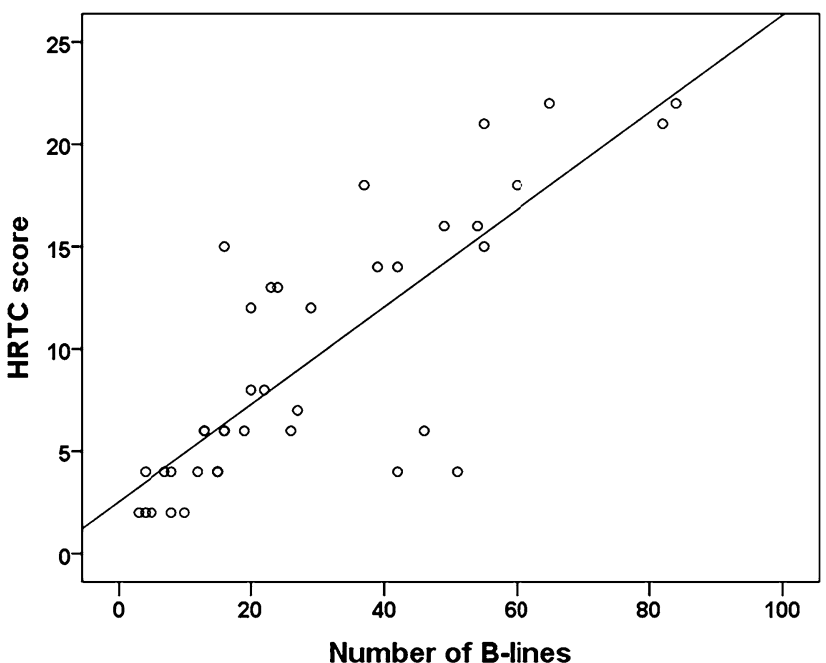

Fig. 1 Correlation between number of B-lines (mean and standard deviation) and high-resolution computed tomography (HRTC) score and carbon monoxide diffusing capacity (DLCO) in 39 patients

determined at a standardized location of 17 different sites of the body through a standardized pinching method, with a score ranging from 0 to 3 [12].

Disease activity and disease severity were measured using European Scleroderma Study Group activity index (DAI) [13] and Medsger scleroderma disease severity scale (DSS) [14], respectively.

\section{Statistical analysis}

The results were expressed as mean and standard deviation (SD) or median and range, as appropriate. Commercial software (SPSS version 22.0) was used for statistical analysis. The coefficient of skewness and the coefficient of kurtosis were used to evaluate the normal distribution of data. Multiple regression analysis was applied to evaluate the relationship between the number of B-lines and the demographic and clinical features (age, duration of disease, mRSS, DAI, DSS). Pearson product-moment correlation coefficient $(r)$ was used to test for an association between numerical variables. Group comparisons were made by Student's unpaired two-tailed $t$ test or the Kruskal-Wallis test, as appropriate. $p$ values $<0.05$ were considered significant.

\section{Results}

The mean number of B-lines are $29.1 \pm 21.8$ and the mean HRCT score is $9.5 \pm 6.4$. PFTs mean values are: DLCO $73.6 \pm 16.5$, FVC $101.7 \pm 18$, FEV1 $98.6 \pm 16$.

A positive correlation exists between the number of B-lines and HRCT score $(r=0.81, p<0.0001)$, conversely there is a negative correlation between the B-lines

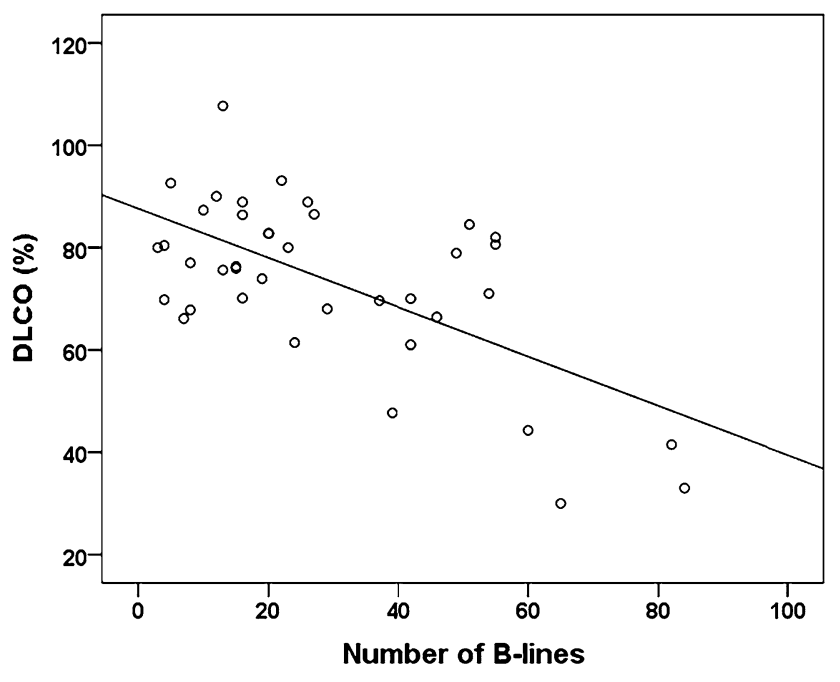

systemic sclerosis patients. The HRTC score was calculated by total Warrick score (mean and standard deviation), the DLCO was expressed as percentage of predicted

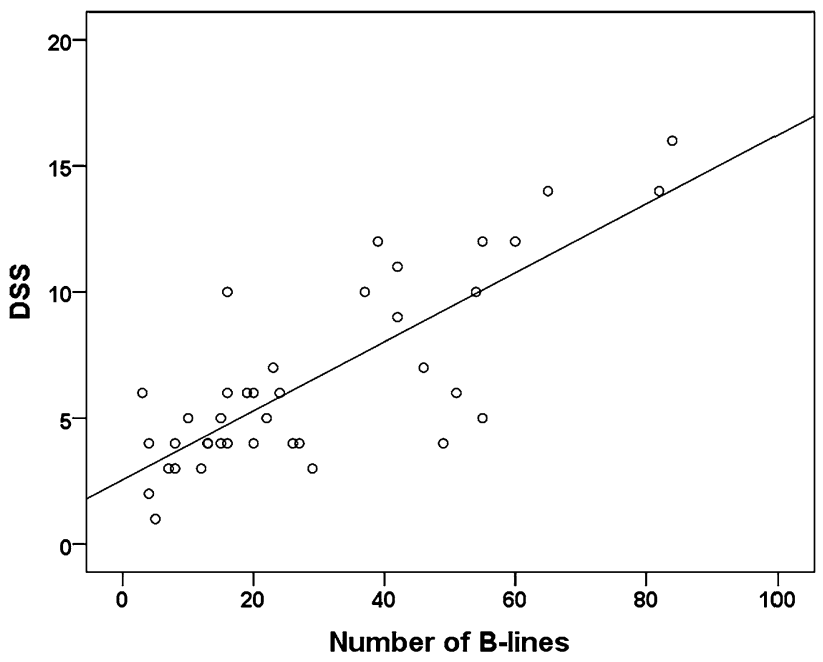

Fig. 2 Correlation between number of B-lines (mean and standard deviation) and disease severity scale (DSS) in 39 patients systemic sclerosis patients. DSS (mean and standard deviation) was calculated by Medsger scleroderma disease severity scale

score, DLCO $(r=-0.63, \quad p<0.0001)$ and FVC $(r=-0.48, p<0.001)$. No significant correlations are found between FEV1 and B-lines number $(r=-0.29$, $p>0.05$ ) (Fig. 1).

In the multiple regression analysis of clinical variables of disease (duration of disease, mRSS, DAI, DSS with age as covariate) only DSS demonstrates a significant correlation with the number of B-lines $(r=0.80, p<0.01)$ (Fig. 2). No significant $(p>0.05)$ correlation is observed with age, disease duration, mRSS, PAPs and DAI. B-lines score are significantly $(p<0.05)$ higher in dcSSc patients than in lcSSc patients [25 (8-84) vs 15 (3-52]. 


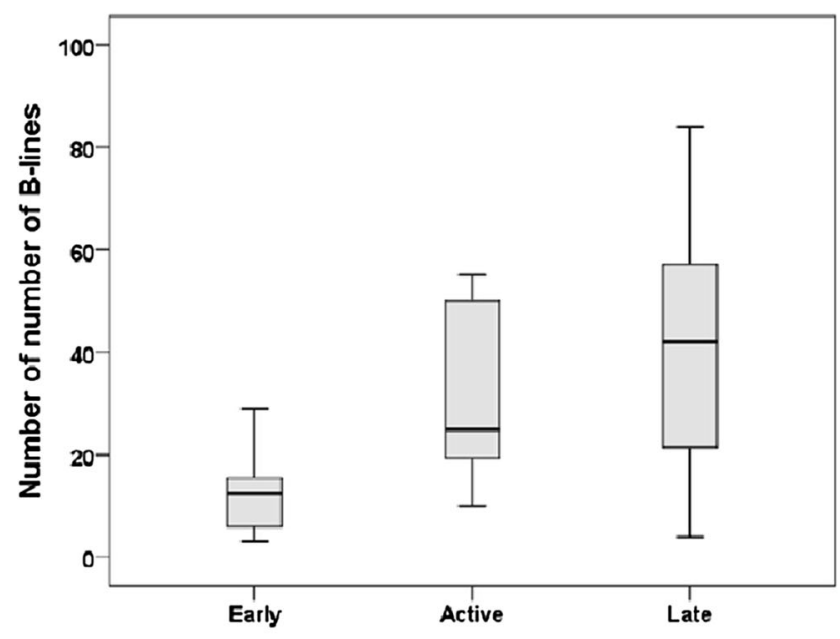

Fig. 3 Number of B-lines (median and range) in 39 patients systemic sclerosis patients grouped by capillaroscopic pattern or digital ulcers history. The left box shows the number of B-lines in $12 \mathrm{SSc}$ patients with early pattern, in 12 with active pattern and 15 with late pattern.

The NVC showed an early capillaroscopic pattern in 12 $(30.8 \%)$ SSc patients, an active capillaroscopic pattern in $12(30.8 \%)$ SSc patients and a late capillaroscopic pattern in $1538.5 \%)$ SSc patients. B-lines scores are significantly $(p<0.01)$ different in the three capillaroscopic groups: early 11.7 (3-29), active 31.1 (10-55), late 41.5 (4-84) (Fig. 3).

Seventeen $(43 \%)$ SSc patients have a digital ulcer history and $22(57 \%)$ SSc patients have no digital ulcer history. In SSc patients with a history of digital ulcer, $s$ the median B-lines score is significantly $(p<0.01)$ higher than in SSc patients with no digital ulcers history [42 (3-84) vs $16(4-55)]$.

\section{Discussion}

Our results confirm that LUS is a very sensitive technique for the evaluation of ILD in SSc patients. The presence of B-lines at LUS examination correlates with HRCT score and DLCO decrease. In particular, we found a positive correlation between B-line numbers and HRCT score, while the number of B-lines negatively correlates with PFTs. LUS has already been used to assess the extent of pulmonary fibrosis in SSc patients. Gargani et al. observed the presence of ultrasound lung comets in about $51 \%$ of SSc patients, and find a significant positive linear correlation between ultrasound lung comets and Warrick scores. The authors conclude that ultrasound lung comets are often present in SSc, more frequently in the diffuse than in the limited form, and that they correlate reasonably well with the HRCT-derived assessment of lung fibrosis [15]. They

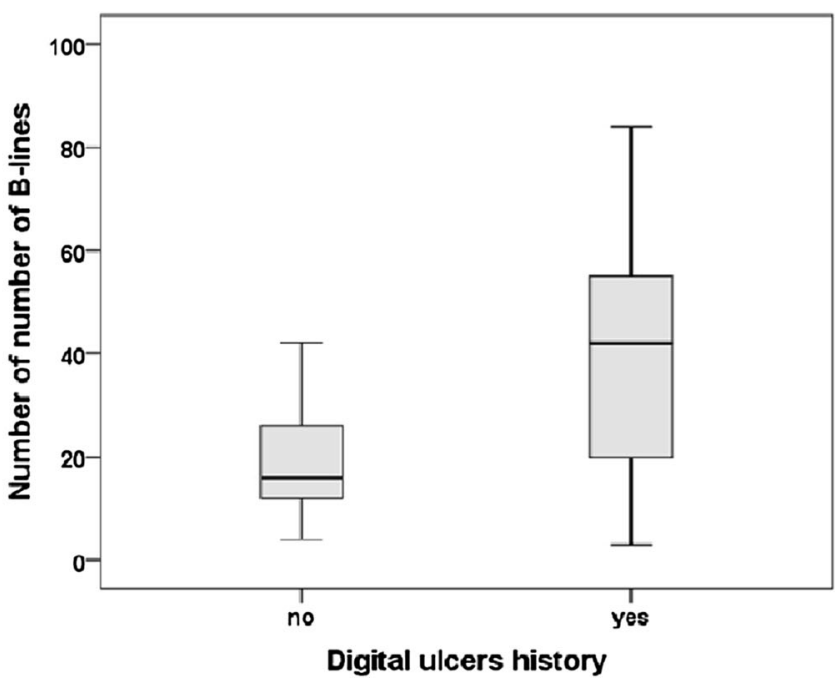

The right box shows the number of B-lines in 17 (43\%) SSc patients with digital ulcers history and in $22(57 \%)$ SSc patients without digital ulcers history

represent a simple, bedside, radiation-free hallmark of pulmonary fibrosis, with a great potential diagnostic and prognostic value. Barskova et al. studied 58 consecutive patients with SSc, finding a significant difference in the number of B-lines between patients with and without ILD at HRCT, with a concordance rate of $83 \%$. Moreover, the authors demonstrate that LUS is a sensitive technique for the evaluation of ILD even in patients with a pretty recent diagnosis of SSc and ILD, finding a positive correlation between B-lines numbers and HRCT score [16]. Other authors evaluated LUS role in other forms of ILD, including primary pulmonary fibrosis and sarcoidosis. Sperandeo et al. in a heterogeneous group of patients with ILD demonstrate that transthoracic ultrasound may document early and late-stage changes associated with the disease [17].

To our knowledge we are the first to postulate that B-lines score positively correlates with DSS and digital vascular damage, evaluated by NVC. The number of $\mathrm{B}$-lines increases with the progression of capillaroscopic damage. In addition, the number of B-lines is significantly higher in SSc patients with DU than in SSc patients without DU. Therefore, we can assume that the number of B-lines increases with the progression of both HRCT score and digital vascular damage. For the first time in this study we demonstrate that in $39 \mathrm{SSc}$ patients the number of B-lines correlates with capillaroscopic and digital vascular damage. Many of the severe internal organ complications of SSc are vascular. Intimal hyperplasia, endothelial dysfunction and occlusive vasculopathy are ubiquitous features of SSc. These vascular lesions are the underlying basis of important clinical syndromes in SSc, including 
Raynaud's phenomenon, digital ulcers, pulmonary arterial hypertension, erectile dysfunction, gastrointestinal complication and renal damage [18]. We propose that vascular damage plays a key role in the pathogenesis of B-lines.

Ultrasound plays a crucial role in the assessment of organ involvement in SSc, and LUS could be an option in association with PFTs for the follow-up of ILD in SSc patients. An important limitation for the use of this technique is that B-lines score lacks specificity, since B-lines can be present in an array of conditions, not only SScrelated ILD. Moreover, in obese patients, or patients with a high thoracic impedance ultrasound is quite difficult to perform.

Future studies could improve the diagnostic detection of LUS using an anterior scanning of the thorax. Nevertheless, although HRCT is the gold standard for the evaluation of ILD, LUS might become a useful tool for guiding the use and timing of HRCT, avoiding for SSc patients a continuous and useless exposure to ionizing radiation.

\section{Compliance with ethical standards}

Conflict of interest The authors declare that they have no conflict of interest.

Statement of human and animal rights The study has been approved by the ethics committee of Sapienza University according to the Declaration of Helsinki.

Informed consent The informed consent was obtained for all individual participants.

\section{References}

1. Shahin AA (2006) Pulmonary involvement in systemic sclerosis. Treat Respir Med 5:429-436

2. Pignone A, Matucci-Cerinic M, Lombardi A et al (1992) High resolution computed tomography in systemic sclerosis. Real diagnostic utilities in the assessment of pulmonary involvement and comparison with other modalities of lung investigation. Clin Rheumatol 11:465-472

3. Diot E, Boissinot E, Asquier E et al (1998) Relationship between abnormalities on high resolution $\mathrm{CT}$ and pulmonary function in systemic sclerosis. Chest 114:1623-1629
4. Porta F, Gargani L, Kaloudi O et al (2012) The new frontiers of ultrasound in the complex world of vasculitides and scleroderma. Rheumatology (Oxford) 51(7):26-30

5. van den Hoogen F, Khanna D, Fransen J et al (2013) Classification criteria for systemic sclerosis: an American College of Rheumatology/European League Against Rheumatism Collaborative Initiative. Arthritis Rheum 65:2737-2747

6. LeRoy EC, Black C, Fleischmajer R et al (1998) Scleroderma (systemic sclerosis): classification, subsets and pathogenesis. J Rheumatol 15:202-205

7. Volpicelli G, Elbarbary M, Blaivas M et al (2012) International evidence-based recommendations for point-of-care lung ultrasound. Intensive Care Med 38:577-591

8. Jambrik Z, Monti S, Coppola V et al (2004) Usefulness of ultrasound lung comets as a nonradiologic sign of extravascular lung water. Am J Cardiol 93:1265-1270

9. Warrick JH, Bhalla M, Schabel SI et al (1991) High resolution computed tomography in early scleroderma lung disease. J Rheumatol 18:1520-1528

10. Miller MR, Crapo R, Hankinson J et al (2005) General considerations for lung function testing. Eur Respir J 26:153-161

11. Cutolo M, Sulli A, Pizzorni C, Accardo S (2000) Nailfold videocapillaroscopy assessment of microvascular damage in systemic sclerosis. J Rheumatol 27:155-160

12. Clements $P$, Lachenbruch P, Siebold J et al (1995) Inter- and intraobserver variability of the total thickness score (modified Rodnan TSS) in systemic sclerosis. J Rheumatol 22:1281-1285

13. Minier T, Nagy Z, Bálint $Z$ et al (2010) Construct validity evaluation of the European Scleroderma Study Group activity index, and investigation of possible new disease activity markers in systemic sclerosis. Rheumatology (Oxford) 49:1133-1145

14. Medsger TA Jr, Silman AJ, Steen VD et al (1999) A disease severity scale for systemic sclerosis: development and testing. J Rheumatol 26:2159-2167

15. Gargani L, Doveri M, D'Errico L et al (2009) Ultrasound lung comets in systemic sclerosis: a chest sonography hallmark of pulmonary interstitial fibrosis. Rheumatology (Oxford) 48:1382-1387

16. Barskova T, Gargani L, Guiducci S et al (2013) Lung ultrasound for the screening of interstitial lung disease in very early systemic sclerosis. Ann Rheum Dis 72(3):390-395

17. Sperandeo M, Varriale A, Sperandeo G et al (2009) Transthoracic ultrasound in the evaluation of pulmonary fibrosis: our experience. Ultrasound Med Biol 35(5):723-729

18. Matucci-Cerinic M, Kahaleh B, Wigley FM (2013) Systemic sclerosis (scleroderma, SSc) is a vascular disease. Arthritis Rheum 65:1953-1962 